\title{
A Bayesian Approach to Estimating Coupling Between Neural Components: Evaluation of the Multiple Component, Event-Related Potential (mcERP) Algorithm
}

\author{
Ankoor S. Shah ${ }^{2}$, Kevin H. Knuth ${ }^{3}$, Wilson A. Truccolo ${ }^{4}$, Mingzhou \\ Ding ${ }^{5}$, Steven L. Bressler ${ }^{5}$, Charles E. Schroeder ${ }^{1,2}$ \\ 'Department of Neuroscience, Albert Einstein College of Medicine, Bronx NY 10461USA \\ ${ }_{2}^{2}$ Cognitive N?uroscience and Schizophrenia Program, Nathan Kline Institute, \\ Orangeburg NY 10962 USA \\ ${ }^{3}$ NASA Ames Research Center, Computational Sciences Department, Code IC \\ Moffett Field CA 94035 USA \\ ${ }^{+}$Neuroscience Department, Brown University, Providence RI 94035 USA \\ ${ }^{5}$ Center for Comflex Systems, Florida Atlantic University, Boca Raton FL 33431 USA
}

\begin{abstract}
Accurate $m$ zasurement of single-trial responses is key to a definitive use of complex electromagnetic and hemodynamic measurements in the investigation of brain dynamics. We developed the multiple component, Event-Related Potential (mcERP) approach to single-trial response estimation 10 improve our resolution of dynamic interactions between neuronal ensembles located in cifferent layers within a cortical region and/or in different cortical regions. The mcERP model ascerts that multiple components defined as stereotypic waveforms comprise the stimulus-evoked response and that these components may vary in amplitude and latency from trial to trial. Maximum a posteriori (MAP) solutions for the model are obtained by iterating a set of equations derived from the posterior probability. Our first goal was to use the mcERP algorithm to analyze interactions (specifically latency and amplitude correlation) between responses in different layers within a cortical region. Thus, we evaluated the model by applying the algorithrı to synthetic data containing two correlated local components and one independent far-field component. Three cases were considered: the local components were correlated by an intercction in their single-trial amplitudes, by an interaction in their single-trial latencies, or by an interaction in both amplitude and latency. We then analyzed the accuracy with which the algorithm estimated the component waveshapes and the single-trial parameters as a function of the linesrity of each of these relationships. Extensions of these analyses to real data are discussed as vell as ongoing work to incorporate more detailed prior information.
\end{abstract}

\section{INTRODUCTION}

Electromagnetic and hemodynamic measurements of brain activity are recorded to investigate neurological processing of sensory stimuli with the assumption that these signals will yield insight into the brain dynamics of sensation. These recorded signals represent the superposition of activity from numerous neural sources on the detector array. Conventionally, the subject is exposed to repeated presentations or trials of the same stimulus, and the recorded signals are averaged across these trials generating the 
average event-related potential (ERP). Although this technique improves signal-toratio, averaging implicitly assumes that an invariant, stereotypical waveform represents the single-irial ERP. This prevents researchers from asking questions about the trial-to-trial variability of the evoked response, about dynamic interactions between stimulus-evcked components, and stimulus-induced modulation of ongoing EEG rhythms.

The multiple component Event-Related Potential (mcERP) model was introduced as an alternative technique for examining these signals. The model states that multiple components generate the signal and that each of these components may vary in amplitude and latency from trial to trial. Furthermore, the mcERP model makes no assumptions about the interdependency of the different components. Thus, this model estimates the different components generated in response to a stimulus presentation, quantifies single-trial variability in the evoked potential (Truccolo 2002), and permits exploration of dynamic interactions between related components. Anatomical studies show connectivity between different cell populations within and between areas, and electrophysiological data based on average responses from awake and anesthetized animals also support these ideas (Schroeder et al. 1998; Nowak and Bullier 1997; Schmolesky et al. 1998). The mcERP model has the potential to show the spatiotemporal profile of activation on a single-trial basis and may reveal information about parallel and ser al processing by sensorineural circuitry.

An interesting bas c circuit suggested by the anatomical and electrophysiological data is the projection from the granular lamina to the supragranular laminae of V1. The granular lamina of V1 (lamina IV) represents the primary thalamorecipient layer of cortex in the visull system, and the supragranular laminae of V1 receive inputs from the granular layer before propagating them to the downstream visual areas. The anatomical and electrophysiological evidence suggests that there may exist a relationship between the amplitudes and latencies of responses simultaneously recorded from these different layers. The mcERP algorithm can address this issue because it estimates the components from the recorded signals and it also estimates their single-trial characteristics. Correlations in the trial-to-trial variability between two components would suggest dynamic coupling between those two neuroelectrophysiological phenomena.

Prior to applicatic $n$ of any new model to real data however, it is helpful to characterize the perfcrmance of the algorithm with a known set of solutions. In this paper, the performance of the mcERP algorithm is evaluated on numerous synthetic data sets consisting of three physiological components. Each data set contains two hypothetical components that display latency coupling, amplitude coupling, or both latency and amplitude coupling. The accuracy of the algorithm in estimating the three hypothetical components is examined by comparing the estimated parameters of the mcERP model with the original parameters utilized in generating the synthetic data. Also, the implications of these simulations are discussed with respect to application of the algorithm to real cata and with respect to possible future refinements of the model. 


\section{METHODS}

\section{The mcERP Model and Algorithm}

The mcERP model (Knuth et al. 2001, Knuth et al. in preparation) describes recorded neuroelectrophysiologic activity as being generated by a set of neural ensembles each responding with a stereotypic activation pattern. The stereotypic activity is a temporal pattern of electrical activity that we will described as a component $s_{n}(t)$, where $s$ is the component's waveshape across time $t$ and $n$ is an index for a particular component. Second, amplitude and latency variability in the evoked response occurs from trial to trial (Truccolo 2002), and the mcERP model modifies the component waveshape $s_{n}(t)$ with a trial-specific amplitude scaling factor $\alpha_{n r}$ and a trial-specific latency shift $\tau_{n r}$, where $n$ still denotes a particular component and $r$ denotes a specific trial. And third, since multiple detectors (referred to as electrodes from now on) are often used, a coupling matrix $C_{m n}$ is added to the model to describe the relation between a particular electrode $m$ and a particular component $n$. This is important since several detectors may record a particular component differentially. These aspects of the racERP model can be written mathematically as:

$$
x_{m r}(t)=\sum_{n=1}^{N} C_{m n} \alpha_{n r} s_{n}\left(t-\tau_{n r}\right)+\eta_{m r}(t)
$$

where $n$ indexes the $N$ components, $C_{m n}$ denotes the coupling between the $m^{\text {ih }}$ detector and the $n^{t h}$ component, $\alpha_{n r}$ is the amplitude scaling of the $n^{t h}$ component during the $r^{t h}$ trial, $\tau_{n r}$ is the latency shift of the $n^{\text {th }}$ component during the $r^{\text {th }}$ trial, $s_{n}(t)$ is the waveshape of the $n^{\text {th }}$ component, and $\eta_{m r}(t)$ is the unpredictable signal recorded in the $m^{\text {th }}$ detector during the $r^{\text {th }}$ trial.

The most probable set of parameters that satisfy the mcERP model expressed in (1) can be calculated from the maximum a posteriori (MAP) solution of the posterior probability. The derivation of the equations governing the most probable parameters for (1) begins with Bcyes' Theorem, which states that the posterior probability

$$
p(\text { model } \mid \text { data }, I)=\frac{p(\text { data } \mid \text { model }, I) p(\text { model } \mid I)}{p(\text { data } \mid I)},
$$

where $p(\cdot)$ is the probability and $I$ is any prior information. The $p$ (data|model, $I$ ) in (2) is the likelihood of the data given the model, the $p($ data $\mid I)$ is called the evidence or the prior probability of the data, $p($ model $\mid \Lambda$ ) is called the prior probability of the model, and the left-hand side of (2) is the called the posterior probability, which is the probability that a given set of model parameters is consistent with the data and the information. Bayes' Theorem describes how the prior probability of the model $p($ model $\mid I)$ is modified by new information such as data. Substituting the parameters of the mcERP model nto (2), the posterior probability becomes

$$
p(\mathbf{C}, \mathbf{s}(t), \boldsymbol{\alpha}, \boldsymbol{\tau} \mid \mathbf{x}(t), I)=\frac{p(\mathbf{x}(t) \mid \mathbf{C}, \mathbf{s}(t), \boldsymbol{\alpha}, \boldsymbol{\tau}, I) p(\mathbf{C}, \mathbf{s}(t), \boldsymbol{\alpha}, \boldsymbol{\tau} \mid I)}{p(\mathbf{x}(t) \mid I)} .
$$


where the bold terms indicate the entire set of a particular parameter. Since the evidence $p(x(t) \mid I)$ is constant as the model parameters vary, the posterior probability can be written

$$
p(\mathbf{C}, \mathbf{s}(t), \boldsymbol{\alpha}, \boldsymbol{\tau} \mid \mathbf{x}(t), I) \propto p(\mathbf{x}(t) \mid \mathbf{C}, \mathbf{s}(t), \boldsymbol{\alpha}, \boldsymbol{\tau}, I) p(\mathbf{C}, \mathbf{s}(t), \boldsymbol{\alpha}, \boldsymbol{\tau} \mid I)
$$

with a proportionalit constant equal to the inverse of the evidence. Next, the prior probability of the moidel can be factored and expressed as the product of the individual probabilities as follows:

$$
\begin{aligned}
& p(\mathbf{C}, \mathbf{s}(t), \boldsymbol{\alpha}, \boldsymbol{\tau} \mid \mathbf{x}(t), I) \propto \\
& \quad p \mathbf{x}(t) \mid \mathbf{C}, \mathbf{s}(t), \boldsymbol{\alpha}, \boldsymbol{\tau}, I) p(\mathbf{C} \mid I) p(\mathbf{s}(t) \mid I) p(\boldsymbol{\alpha} \mid I) p(\boldsymbol{\tau} \mid I)
\end{aligned}
$$

The $p(\alpha \mid I)$ and the $p(\tau \mid I)$ are assigned uniform distributions and given ranges that are physiologically realizable. A Gaussian likelihood is assigned to (5) based on the principle of maximum entropy (Sivia, 1996; Jaynes, unpublished) resulting in

$$
\begin{aligned}
& p(\mathbf{C}, \mathbf{s}(t), \boldsymbol{\alpha}, \boldsymbol{\tau}, \sigma \mid \mathbf{x}(t), I) \propto \\
&\left(2 \pi \sigma^{2}\right)^{\frac{-M R T}{2}} \operatorname{Exp}\left[-\frac{1}{2 \sigma^{2}} Q\right] p(\sigma \mid I) p(\mathbf{C} \mid I) p(\mathbf{s} \mid I)
\end{aligned}
$$

where $\sigma$ is the expec ed variance between the predictions and the mean, $p(\sigma \mid I)$ is the prior probability of $\sigma$, and $Q$ represents the sum of the square of the residual between the data and model. $Q$ is derived from (1) as

$$
Q=\sum_{m=1}^{M} \sum_{r=1}^{R} \sum_{t=1}^{T}\left(x_{m r}(t)-\sum_{n=1}^{N} C_{m n} \alpha_{n r} s_{n}\left(t-\tau_{n r}\right)\right)^{2},
$$

where $M$ is the tota number of detectors or electrodes, $R$ is the number of trial presentations of a stimulus, $T$ is the number of the data points sampled. A Jeffrey's prior is assigned to $\sigma$ such that $p(\sigma \mid I)=\sigma^{-1}$, and the joint posterior probability of $(6)$ is marginalized over all values of $\sigma$ giving the marginal joint posterior probability

$$
p \mathbf{C}, \mathbf{s}(t), \boldsymbol{\alpha}, \boldsymbol{\tau} \mid \mathbf{x}(t), I) \propto Q^{\frac{-\Delta / R T}{2}} p(\mathbf{C} \mid I) p(\mathbf{s} \mid I) .
$$

The derivation continues with the assignment of prior probability for the coupling matrix and the component waveshapes. The solution of the electromagnetic forward problem could be used to generate knowledge about the source-detector coupling (Knuth, 1998; Knutl \& Vaughan, 1999), but for simplicity only uniform prior probabilities were assigned to $p(C \mid I)$ and $p(s \mid I)$. When is this done and when the natural logarithm is taken, the marginal joint posterior probability becomes

$$
\ln P=\frac{-M R T}{2} \ln Q+\text { const },
$$

where $\mathrm{P}$ is $p(\boldsymbol{C}, \boldsymbol{s}(t), \alpha, \tau \mid \boldsymbol{x}(t), I)$.

The most probable set of parameters for (9) are then determined by solving the expression for the maximum a posteriori (MAP) solution of the posterior probability by setting the derivat ve of the posterior with respect to each of the model parameters to zero. The resulting equations are not shown here but are found in Knuth et al. 
(2001, in preparatio 1 ). These equations are then incorporated into an iterative algorithm that initially assumes the existence of a single component within the data and refines the parameters $s_{J}(t), \alpha_{l r}, \tau_{l r}$, and $C_{m l}$ until there is less than $1 \%$ change in the waveshape of the $s_{l}(t)$ or until 15 iterations are complete. After estimating a single component, the residual signal is calculated as

$$
\text { residual }_{m}=x_{m r}(t)-\sum_{n=1}^{N} C_{m n} \alpha_{n r} s_{n}\left(t-\tau_{n r}\right)
$$

If the residual displays structure across trials, the mcERP algorithm is applied again to estimate a second component $s_{2}(t)$ and its related parameters. In this second set of iterations, both $s_{J}(t), s_{2}(t)$, and their related parameters are updated. This process of adding components continues until the user determines that the residual signal contains no structure across trials.

\section{The Synthetic Data}

Synthetic data were created to evaluate the performance of the algorithm on data containing two interacting neural sources. The structure of the synthetic data simulated field potertial recordings from a linear, 15-channel multielectrode array spanning laminae 1 through 6 of macaque Vl during presentation of a red flash of light. In each simulated case, 50 trials of simulated data for each of the 15 channels of the electrode array were generated. Three separate components were specified (Figure 1A): (i) Component 1 resembled activation of a spiny stellate cell population in
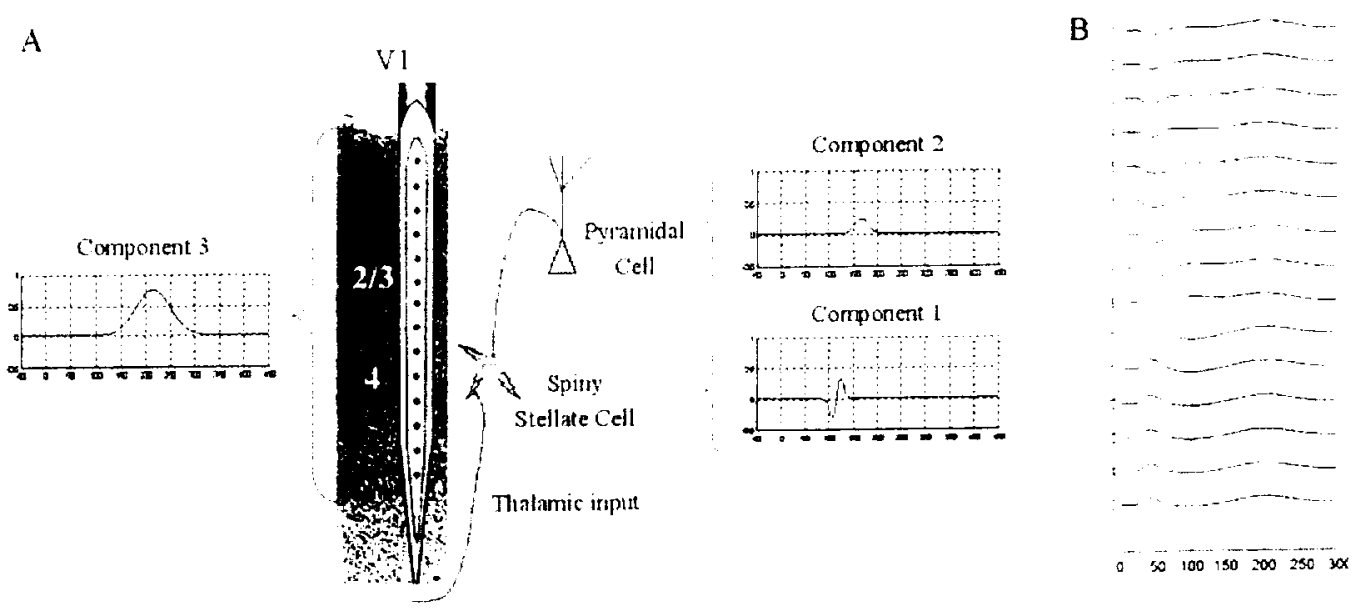

Figure 1. Synthetic data created to evaluate the mcERP algorithm was based on field potential recordings from a linear, nultielectrode array positioned to span laminae 1 through 6 simultaneously. A. Component 1 of the synthetic data represents activation of the lamina 4 spiny stellate cells by a thalamic input. Compone it 2 represents the subsequent activation of pyramidal cells in the lamina 2 or 3 , and component 3 is a car-field source whose component is recorded approximately equally by all electrode channels. B. The spatial distribution of the three components is observed in their linear summation at every electrinde channel. Component 1 is located between channels 9 and 10 , component 2 is located between chanels 4 and 5 , and component 3 is seen nearly equally by each electrode channel. 
granular lamina 4 by a thalamic input; (ii) Component 2 represented activation of pyramidal cells in sup ragranular lamina 2 or 3 by a granular input such as component 1 ; and (iii) Componert 3 is the electrical activity generated by a far-field source. The temporal patterns of these components were chosen to resemble signals observed in $\mathrm{V} 1$ in response to a red flash of light. A coupling matrix specified the relative positioning of the different sources such that component 1 was located between channels 9 and 10, component 2 was located between channels 4 and 5, and component 3 was seen nearly equally across the channels of the electrode array (Figure 1B). Low-frequency noise with a spectrum of $1 / \mathrm{f}$ was added to the data, and the standard deviatior: of the noise was specified as 0.063 so that the signal-to-noise ratio (SNR) of the smallest component (component 2) was 1.1 as given by $S N R=\sigma_{\text {componen }}^{2} / \sigma_{\text {noise }}^{2}$ where $\sigma$ denotes the standard deviation across time.

Components 1 and 2 of the synthetic data were coupled so that component 1 drives component 2 via a feedforward connection. We studied four different implementations of such a relationship. Method 1 specified a relationship between the latency shifts of components 1 and 2 so that when the first component was delayed, so was the second, and when the first component was early, so was the second. The degree to which the sources were coupled was controlled by a coupling parameter $k$ (not to be confused with the coupling matrix). When $k=0$, the sources are uncoupled and the latency of component 2 jitters independent of component 1 . When $k=1$, the sources are completely coupled so that component 2 follows component 1 precisely. This is written in equation form as

$$
\tau_{2 r}=(1-k) N\left(0, \sigma_{\text {jlat }}\right)+k \tau_{1 r},
$$

where $\tau_{/ r}$ and $\tau_{2 r}$ are the latency shifts of the first and second components respectively in the $r^{t h}$ trial, $k$ is a constant that varies the coupling between the components, $N\left(0, \sigma_{j l a t}\right)$ represents a random number sampled from a normal distribution with mean equal to 0 and standard deviation equal to $\sigma_{j l a t}$, and $\tau_{j r}$ is the latency shift of the first component in the $r^{\text {th }}$ trial. Single-trial synthetic data were generated for the 15 electrode channels during 50 trials of the stimulus. The amplitude scaling factors of the three components in each trial were independent of each other and defined from a randomly sampled lognormal distribution with a sample mean $\mu_{a m p}=1.0$ and sample standard deviation $\sigma_{a n p}=1.0$. The latency shifts of component 1 and component 3 were independent and defined from a normal distribution $N(0,10.0$ milliseconds $)$ with sample mean $\mu_{\text {lat }}=0.0$ milliseconds and sample standard deviation $\sigma_{\text {lat }}=10.0$ milliseconds. Eleven separate cases were explored with $k=\{0.0,0.1, \ldots, 1.0\}$. In each case, $\sigma_{\text {jlat }}$ was equal to 10.0 milliseconds.

Method 2 specifiet a coupling between the amplitudes of components 1 and 2 . This relation was given by:

$$
\alpha_{2 r}=(1-k) \times 2^{N\left(1, \sigma_{\text {,amp }}\right)}+k \frac{2}{1+e^{-b\left(\alpha_{1 r}-1\right)}},
$$

where $\alpha_{I r}$ and $\alpha_{2 r}$ are the amplitude scaling factors of the first and second components respectively in the $r^{\text {th }}$ trial, $k$ is a constant that varies the coupling, $N\left(l, \sigma_{\text {jamp }}\right)$ represents a random rumber from a normal distribution with sample mean equal to 0 
and sample standard cieviation equal to $\sigma_{j a m p}$, and $\alpha_{l r}$ is the amplitude scaling factor of the first component in the $r^{\text {th }}$ trial. The first term in the sum on the right-hand side of (12) represents the independent lognormal amplitude jitter of component 2, whereas the second term represents a sigmoid function whose rise is varied by $b$ (Figure 2 ). As in Method 1, single-trial data for 15 electrode channels were generated across 50 trials. The latency shifts of the three components were independent and chosen randomly from $N(0,10.0$ milliseconds). The amplitude scaling factors of components 1 and 3 were also independen: and chosen from a lognormal distribution with a sample mean $\mu_{a m p}=1.0$ and sample standard deviation $\sigma_{a m p}=1.0$. Forty different simulations were performed using this method with $\sigma_{\text {jamp }}=0.75, b=\left\{2^{0}, 2^{1}, 2^{2}, 2^{3}\right\}$, and $k=\{0.1,0.2$, $\ldots, 1.0\}$ for each value of $b$. Since a negative value for $\alpha$ is not physiologically realizable, each $\alpha_{2 r}$ value was adjusted by $\left(1-<\alpha_{2}>\right)$, where $\alpha_{2}$ represents all amplitude scaling factors across $R$ trials and $<>$ represents the mean of that sample.

Method 3 combined elements of the two previous methods so that the components were coupled both by latency shifts and amplitude scaling factors. The latency and amplitude relations were specified by (11) and (12) respectively, and the single-trial data were again generiated for 15 electrode channels during 50 trial presentations of the stimulus with the single-trial amplitudes and latencies of components 1 and 3 determined as before. The latency and amplitude relationships between components 1 and 2 were calculated with $\sigma_{\text {jlat }}=10.0$ milliseconds, $\sigma_{\text {jamp }}=0.75, b=\left\{2^{-4}, 2^{-3}, 2^{-2}, 2^{-1}\right.$, $\left.2^{0}, 2^{2}, 2^{3}\right\}$, and $k=\{9.0,0.1, \ldots, 1.0\}$ for each value of $b$ resulting in 77 simulated cases.

Finally, Method 4 ivas identical to Method 3 except that the stereotypic waveshape of component 2 was altered to be identical to component 1 . The latency and

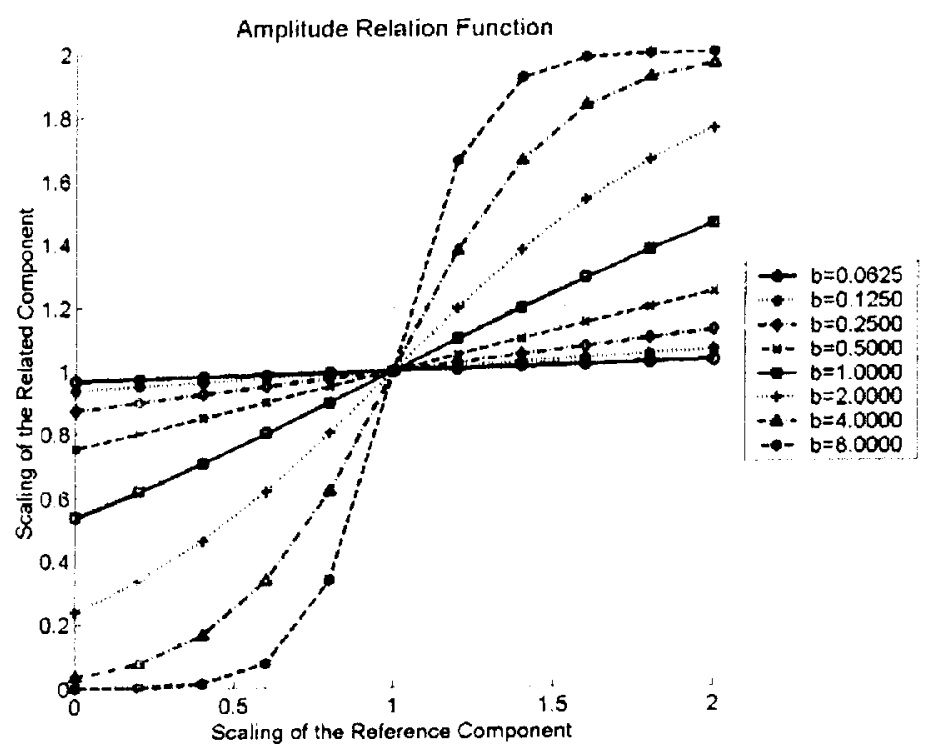

Figure 2. Amplitude relation function used in the simulated cases studying amplitude relation alone and those studying simultaneous amplitude and latency relation. As the value of $b$ is increased, the relation varies from linear to sigmo dal. 
amplitude relations were specified with $\sigma_{\text {jlat }}=10.0$ milliseconds, $\sigma_{\text {jamp }}=0.75, b=\left\{2^{-3}\right.$, $\left.2^{-1}, 2^{0}, 2^{1}, 2^{2}, 2^{3}\right\}$, and $k=\{0.0,0.1, \ldots, 1.0\}$ for each value of $b$. Thus sixty-six different combinations were simulated.

\section{Evaluation of Algorithm Performance}

The mcERP algorithm performance was evaluated with several different measures of accuracy. First, the Amari error (Amari et al. 1996) was calculated to quantify the algorithm's ability tc separate each component comprising the synthetic data. The basis of this error is derived from the linear mixing model given by

$$
x=C s \text {, }
$$

where $x$ is a matrix of the signals recorded in each detector across time, $C$ is the mixing (or coupling) matrix describing the component-detector relationship, and $s$ is a matrix of the component waveshapes across time. The linear mixing model of (13) possesses two indeterminacies because the coupling coefficients in matrix $C$ and the component waveshap is in matrix $s$ can be rescaled by a diagonal scaling matrix $\Sigma$

$$
x=C \Sigma \Sigma^{-1} s
$$

and the order of the sources can arbitrarily be changed by a permutation matrix $\Pi$

$$
x=C \Sigma \Pi \Pi^{-1} \Sigma^{-1} s .
$$

Thus, the linear mixing model can be rewritten as

$$
x=(C \Sigma \Pi)\left(\Pi^{-1} \Sigma^{-1} s\right)
$$

Expression (16) asserts that estimates of the coupling matrix and the component waveshapes will be $s \mathrm{~s}$ accurate as a scaled permutation of the original and may be written as

$$
\hat{s}=M^{-1} s .
$$

or

$$
\hat{C}=C M \text {. }
$$

where $M$ is a matrix describing the relation between the original matrix and the estimated version. A perfect estimate of the coupling matrix or the component waveshapes would result in $M$ being a scaled permutation matrix equivalent to $M=\Sigma \Pi$. Thus, studying $M$ yields insight into the accuracy of the estimated quantity. Solving for $M^{\prime}$ in (17) gives

$$
M^{-1}=\left(\hat{s} s^{T}\right)\left(s s^{T}\right)^{-1}
$$

and in (18) yields

$$
M^{-1}=\left(\left(C^{T} C\right)^{-1}\left(C^{T} \hat{C}\right)\right)^{-1}=\left(C^{T} \hat{C}\right)^{-1}\left(C^{T} C\right),
$$

where $T$ denotes the transpose of the marked matrix. The deviation of $M^{\prime}$ from a scaled permutation matrix is found by the computing the Amari error 


$$
E_{A \text { i ari }}=\frac{\sum_{i=1}^{n}\left(\frac{\sum_{j=1}^{n}\left|A_{i j}\right|}{\max _{k}\left|A_{i k}\right|}-1\right)+\sum_{j=1}^{n}\left(\frac{\sum_{i=1}^{n}\left|A_{i j}\right|}{\max _{k}\left|A_{k j}\right|}-1\right)}{2\left(n^{2}-n\right)},
$$

where $A$ we set equal to $M^{\prime}, i$ indexes the rows of matrix $A, j$ indexes the columns of matrix $A, n$ is the total number of components, $k$ is the index of the position of the maximal value, and the denominator normalizes the error. Either (19) or (20) can be used to calculate the Amari error; (19) was used to calculate the Amari error for relationship methods 1,2 , and 3 , and (20) was used to evaluate the Amari error in relationship method 4 because the quantity $\left(s s^{T}\right)^{-1}$ is singular when components 1 and 2 have the same wave shape.

Comparing the estimated component waveshapes to the actual component waveshapes was the iecond tool used to evaluate the performance of the algorithm. The root-mean-square (RMS) error was calculated as follows:

$$
E_{\text {wave }}^{j}=\frac{\sqrt{\sum_{t=1}^{T}\left(s_{j}(t)-\hat{s}_{j}^{\prime}(t)\right)^{2}}}{\sqrt{\sum_{t=1}^{T}\left(s_{j}(t)\right)^{2}}},
$$

where $j$ indexes a particular component, $T$ is the total number of time samples, and $\hat{s}^{\prime}$ denotes the estimated sources $\hat{s}$ after they have been scaled and permuted so as to be comparable to the original sources.

Accuracy of the latency shifts and amplitude scaling factors was examined by calculating the average absolute difference (average ABS error) between the original values and the estimated ones. The following two equations define these errors for each component $j$ acrcss all $R$ trials:

$$
\begin{aligned}
E_{\text {amp }}^{j} & =\frac{1}{R} \sum_{r=1}^{R}\left|\alpha_{j r}-\hat{\alpha}_{j r}\right| ; \\
E_{\text {lat }}^{j} & =\frac{1}{R} \sum_{r=1}^{R}\left|\tau_{j r}-\hat{\tau}_{j r}\right| .
\end{aligned}
$$

ABS errors below the standard deviation of the variability $\left(\sigma_{\text {jamp }}=1.0\right.$ and $\sigma_{\text {jlat }}=10.0$ milliseconds) signify an improvement in estimation over that of standard averaging.

\section{RESULTS}

Performance was first evaluated for synthetic data generated using coupling method 1. Each measure of performance showed that strong latency coupling does not hamper mcERP's ability to ejtimate the parameters. For each value of $k$, the Amari error (Figure $3 \mathrm{~A}$ ) remains below 0.06 , which indicates less than about $1 \%$ error in 

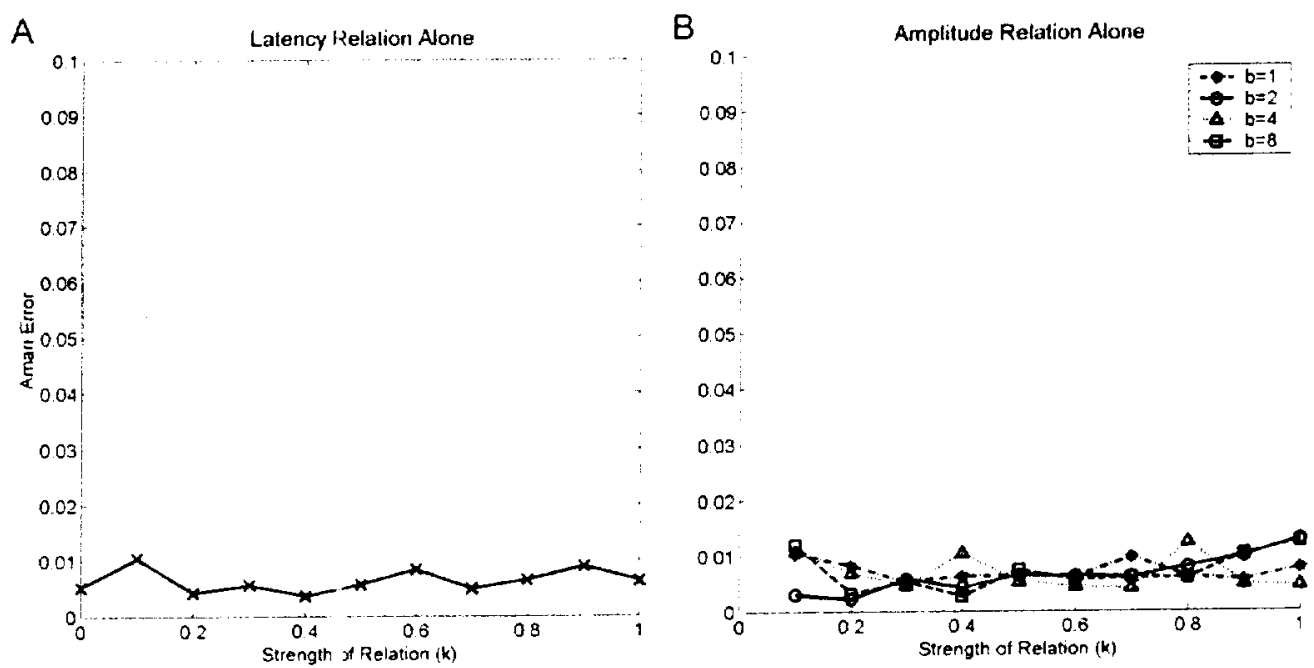

Figure 3. The resulting Imari error when the mcERP algorithm is applied to synthetic data with a latency relation alone $(\mathrm{A})$ and with an amplitude relation alone $(\mathrm{B})$.

separation. Although not shown, the component RMS error is below $10 \%$ and does not illustrate a trend with increasing coupling. Finally the average absolute errors of the amplitude scaling factors and the latency shifts are well below the standard deviation of the original data.

Method 2 related the amplitude of component 2 to the amplitude of component 1 via a sigmoid function (12) with a parameter $b$, which controls the slope of the transition (larger $b$ gives a larger slope). Both the transition parameter $b$ (Figure 2) and the coupling constant $k$ were varied. Figure 3B illustrates the Amari error for method 2. Again the Amari errors were relatively constant as a function of $b$ and $k$, and were less than 0.06 . The other parameters were also well estimated.

Method 3 combined the aspects of methods 1 and 2 such that simultaneous amplitude and latency relations were considered. Again, the mcERP algorithm estimated the components and their single-trial parameters accurately. Figure 4A shows that the Amari error does not vary across $k$ and $b$.

The final test of the mcERP algorithm studied synthetic data whose component 1 and component 2 had the identical waveshapes and were related both in an amplitude and latency. Table 1 lists the Amari errors for all of the different cases considered in this block of simulations; there are only two cases where the Amari error falls below 0.06 indicating separation quality was poor in most cases. Figure $4 \mathrm{~B}$ illustrates a surface plot of these values, and shows that as strength of the interaction $k$ increases, the Amari error also ncreases. In contrast, as the amplitude interaction varies from linear (small $b$ ) one iigmoid-shaped (large $b$ ), the Amari error does not follow an appreciable pattern. Examining the component waveshape, amplitude scaling factor, and latency shift errors (all not shown), several different relationships were observed.

First, component 1 waveshape error increased with increasing $k$ while remaining relatively constant with varying $b$. Component 1 's estimated amplitude scaling factor 
A

Related Wavesha es are Different

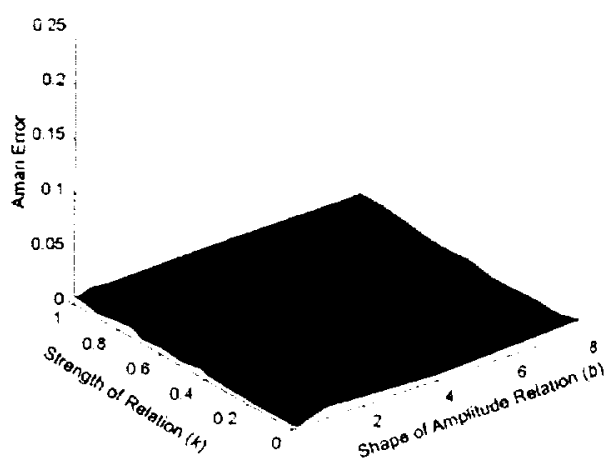

B

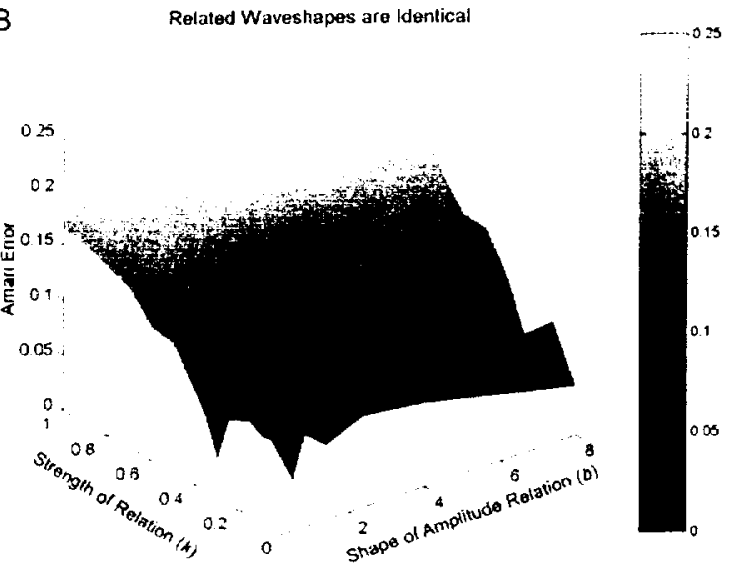

Figure 4. mcERP algorith $n$ performance on synthetic data containing both an amplitude and a latency relation between components 1 and 2. Panel A shows the Amari error when the related components' waveshapes differed, and II shows the Amari error when the components' waveshapes were identical.

error was largest wht $\mathrm{n}$ both $k$ and $b$ were small, and estimated latency shift error decreased with increasing $k$ while remaining constant with varying $b$. Second, component 2's RMS waveshape error, average ABS amplitude scaling factor error, and average ABS latency shift error all increased with increasing $k$ and all increased slightly with increasing $b$. Finally, the accuracies of component 3 estimates (not shown) exhibit no re ation to both $k$ and $b$ and were equivalent to those in other simulations for all practical purposes.

Samples of the restalts for two different cases are shown in Figure 5 and Figure 6. Figure $5 \mathrm{~A}-\mathrm{C}$ illustrates an example from method 3 where the waveshapes of components are different, and where $b=8.0$ and $k=1.0$. The estimated component waveshapes, their spat al distributions, amplitude scaling factors, and latency shifts are shown with respect to their original counterparts. Figure 6A-C shows a case from relation method 4 where the waveshapes of components 1 and 2 were identical, and where $b=8.0$ and $k=1.0$. In this case, the component waveshapes and their spatial

Table 1. Amari error whe' the mcERP algorithm is applied to synthetic data where components 1 and 2 have the same waveshape and an amplitude and latency relation.

\begin{tabular}{c|cccccc}
\hline \multirow{2}{*}{$k$ value } & \multicolumn{7}{c}{$b$ value } \\
\cline { 2 - 7 } & 0.2500 & 0.50 & 1.00 & 2.00 & 4.00 & 8.00 \\
\hline 0.0 & 0.0934 & 0.0461 & 0.1015 & 0.0835 & 0.0749 & 0.0438 \\
0.1 & 0.0773 & 0.0714 & 0.0765 & 0.0491 & 0.0790 & 0.0923 \\
0.2 & 0.1081 & 0.0771 & 0.0942 & 0.0888 & 0.0966 & 0.0482 \\
0.3 & 0.0350 & 0.0662 & 0.0738 & 0.0744 & 0.0746 & 0.1077 \\
0.4 & 0.0931 & 0.0605 & 0.1075 & 0.1205 & 0.1037 & 0.1431 \\
0.5 & 0.1160 & 0.0949 & 0.0930 & 0.1407 & 0.1379 & 0.1452 \\
0.6 & 0.1200 & 0.1286 & 0.1399 & 0.1226 & 0.1360 & 0.1742 \\
0.7 & 0.1431 & 0.1574 & 0.1737 & 0.1662 & 0.1840 & 0.2227 \\
0.8 & 0.1521 & 0.1664 & 0.1848 & 0.1805 & 0.2104 & 0.2241 \\
0.9 & 0.1593 & 0.1828 & 0.1968 & 0.2098 & 0.2360 & 0.2350 \\
1.0 & 0.1641 & 0.1908 & 0.2112 & 0.2342 & 0.2428 & 0.2298
\end{tabular}


distributions are not well estimated because the components are not separated. In addition, method 4 results in greater errors in the estimates of the single-trial features as observed in Figure $6 \mathrm{~B}$ and $\mathrm{C}$ in comparison to those illustrated in Figure $5 \mathrm{~B}$ and $\mathrm{C}$.

\section{DISCUSSION}

The mcERP model describes the single-trial event-related potential as the linear summation of multip e components each described by a stereotypic waveshape and possessing a trial-specific amplitude and latency. Application of Bayes' theorem to the model allows development of an algorithm that gives the most probable set of parameters to satisfy the model. The advantage of utilizing Bayes' theorem is that algorithm failure results only from model inadequacies, inappropriate or insufficient prior information abcut the situation, or insufficient data. In many cases, model inadequacies may be identified by testing the algorithm on synthetic data prior to application to real dati.

The performance of the algorithm on data with interacting components is critical as dynamical coupling between real cortical components is expected. Furthermore, such coupling between components will yield insights into sensory processing within and between cortical areas In this paper, the mcERP algorithm was tested using numerous synthetic data sets that reconstruct four hypothetical relations between granular and supragranular components when evoked by an external stimulus. Amplitudeamplitude and latency-latency interactions between two components were found not to affect the ability of the algorithm to identify the component waveshapes, their spatial locations, or their single-trial amplitude and latency characteristics. These latencylatency results were expected as previous studies of the mcERP algorithm found that accurate identification of three components with independent amplitude variability but no latency variability was possible (Knuth et al. in preparation). This is because a lack of latency variability in the components can be described by (11) when $\tau_{/ r}=0$ and $k=$ 1.

The second set of simulations explored an amplitude interaction between two components. A sigmoid relation was utilized because it provides a threshold and saturation. The threshold of the sigmoid relation prevents occurrence of the driven component when the driving component's activation level is below a given threshold. The saturation of the sigmoid curve prevents a further increase in the driven component's amplitude when all elements of the neuronal ensemble generating this component are "recrtited." In this set of simulations, the algorithm accurately estimated all model parameters.

Simultaneous amplitude and latency interactions between the granular and the supragranular components were examined in the third group of simulations. Again, the mcERP algorithm accurately estimated all parameters. We hypothesized that even though the single-trial characteristics of components 1 and 2 were related, mcERP succeeded in identification due to the fact that the waveshapes of the components were different. To test this hypothesis, we performed the fourth set of simulations where the waveshapes of the coupled components were identical. The quality of separation, 

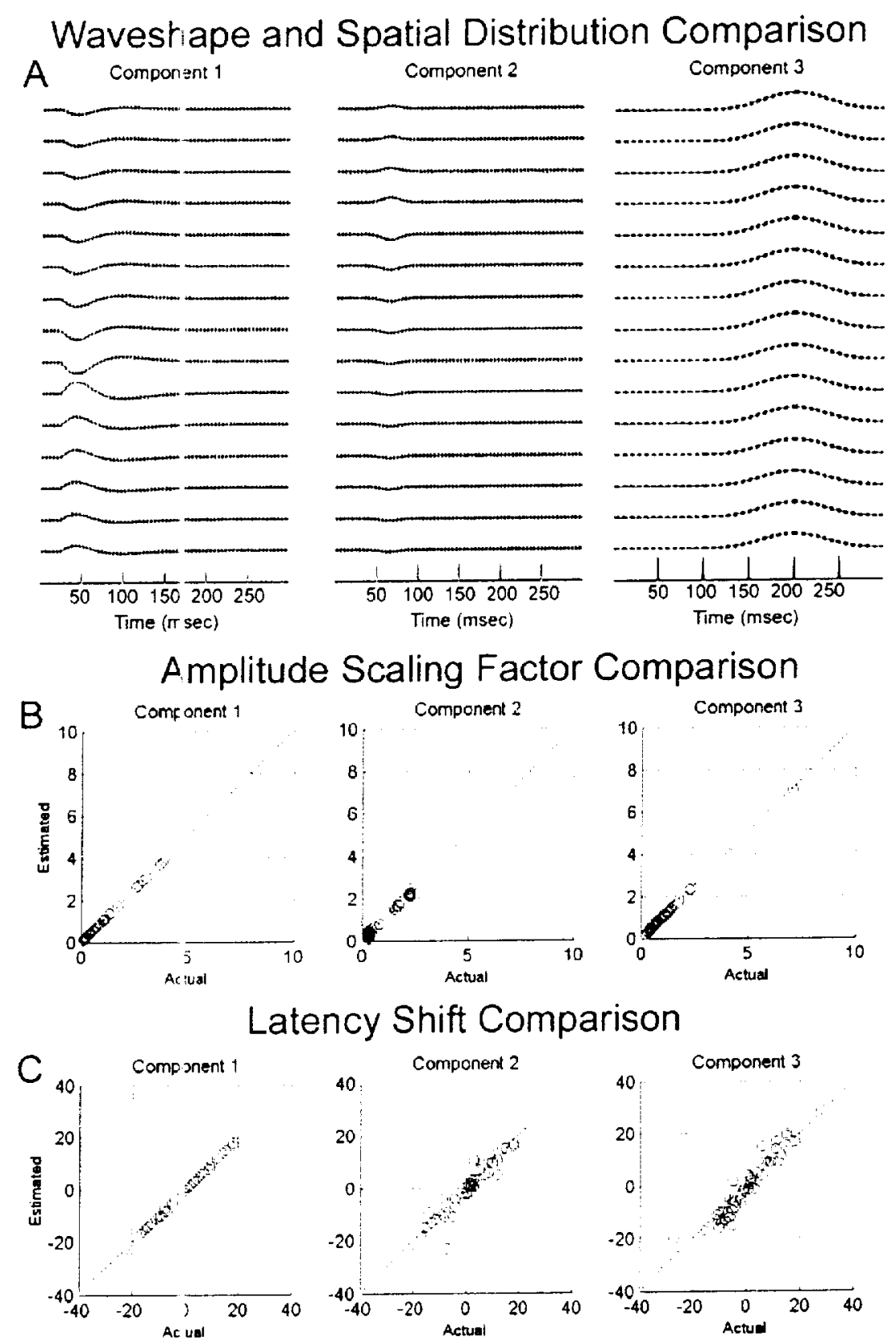

Figure 5. mcERP algorithm estimates match the original synthetic data well. In this case, the waveshapes of component 31 and 2 are different, $k=1.0$, and $b=8.0$. A. The component waveshapes and their spatial distribut ons are plotted across electrode channels. The dotted lines in each plot indicate the estimated con:ponent, and the solid lines indicate the original waveshapes. The blue lines are difficult to observe because the estimated components match the original ones well. B. Comparison of the actual and estimated amplitude scaling factors. C. Comparison of the actual and estimated latency timeshits. The dotted diagonal line denotes a perfect match between the actual and the estimated values in bot $B$ and $C$. 


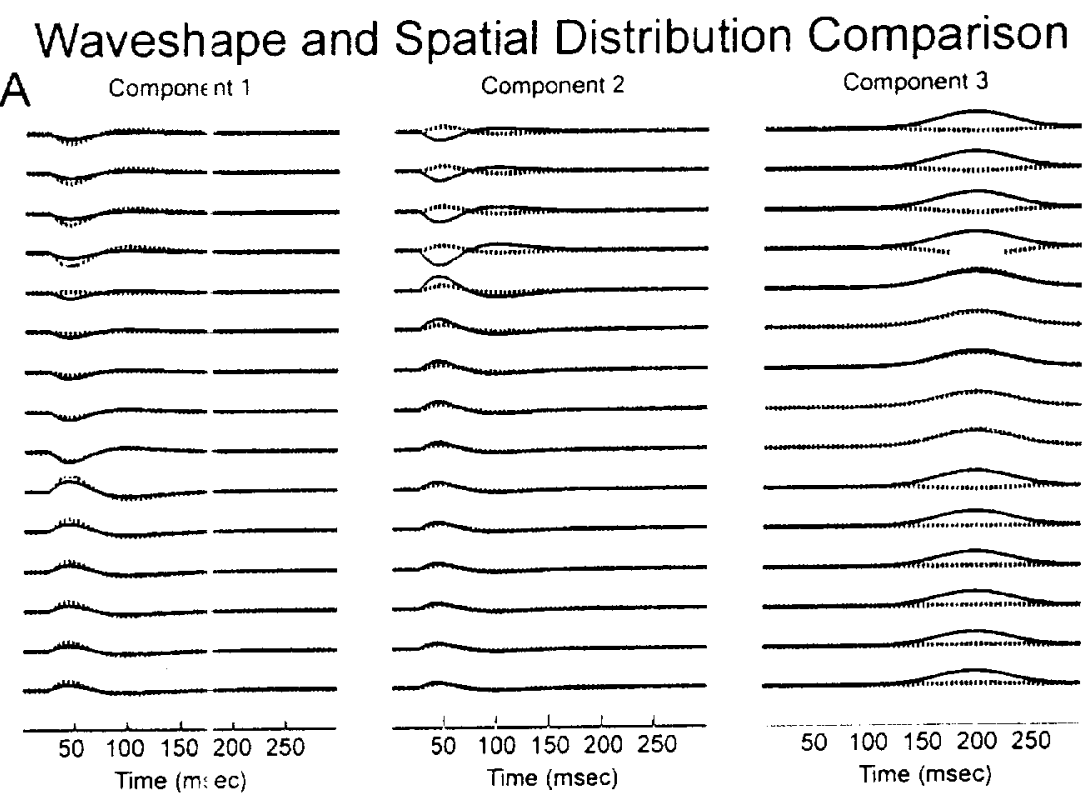

Amplitude Scaling Factor Comparison
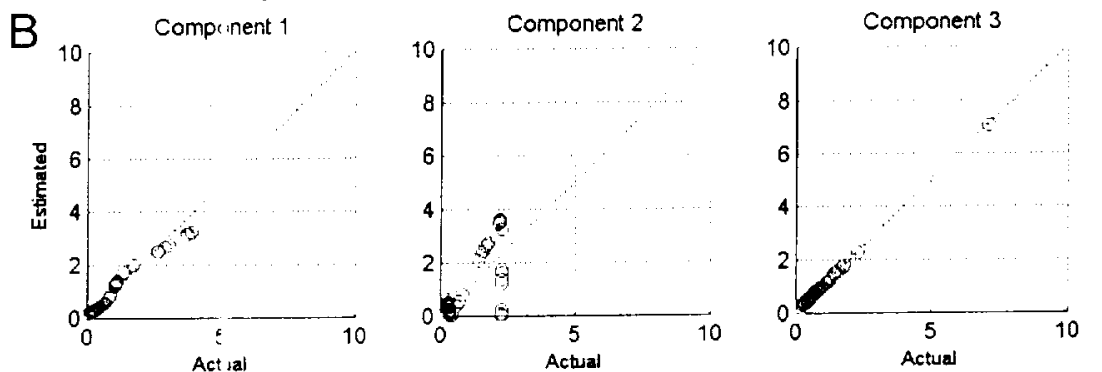

\section{Latency Shift Comparison}
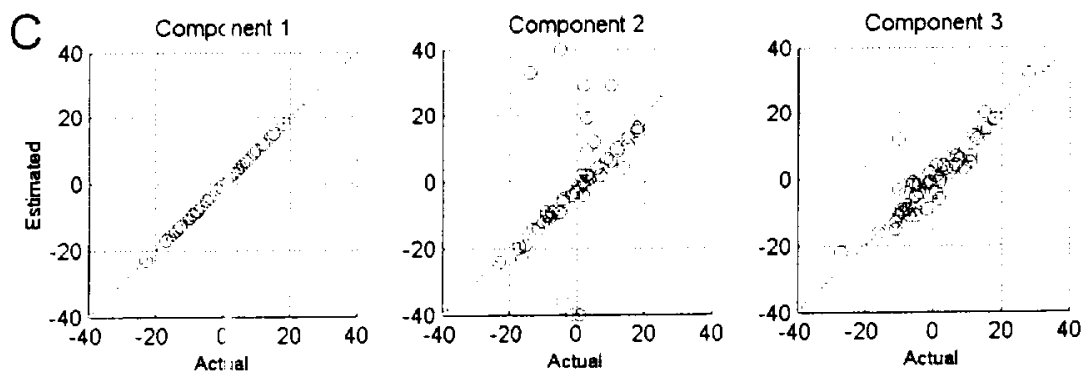

Figure 6. mcERP algoritl im estimates do not match the original synthetic data well. In this particular case, the waveshapes of components 1 and 2 are equivalent, $k=1.0$, and $b=8.0$. A. The component waveshapes and their spatial distributions are plotted across electrode channels. The dashed lines indicate the estimated component, and the solid lines indicate the original waveshapes. B. Comparison of the actual and estimated amplitude scaling factors. C. Comparison of the actual and estimated latency timeshifts. The dotted, diagonal line denotes a perfect match between the actual and the estimated values in both $B$ and $C$. 
as measured by the Amari error, degrades as the strength of their interaction increases but remains relatively unaffected by changes in the shape of the amplitude relation. The amplitude scaling factor error and the latency shift error show an inverse relationship between the two related components. Since there is considerable mixing between these two components, this observation may indicate that the first of the two related components estimated by the algorithm is better approximated than the second. Indeed, the waveshapes for the highly coupled case in Figure 6 suggest that this is the case. Together these findings indicate that a difference in the temporal activation pattern of the component is crucial in separating strongly interacting components. When the component waveshapes are the same, independent variation of the amplitude and latency parameters of the components can assist the algorithm in separating the components, but this is not typically sufficient to accurately estimate all of the parameters.

Although several scenarios were considered, other interactions are possible. For example, amplitude-atency couplings may exist so that a larger than average amplitude of a component in a given trial may result in the earlier activation of a second, dynamically coupled component. Investigating the effect of the spatial distribution on the estimation of two dynamically coupled sources may comprise a second study. Last, the degree to which differences in the waveshapes affect the quality of separation might be explored.

By applying the m:ERP algorithm to real data we expect to be able to characterize the single-trial properties of these evoked responses, which will provide valuable insights into the cortical processing of sensory stimuli. The results of the simulations presented here suggest that the mcERP algorithm will be able to distinguish between spatially distinct neuril ensembles generating differing component waveshapes despite the fact that they may be interacting with one another. However, as the waveshapes of the two components become more similar, we expect the quality of separation to degrade. This scenario may be addressed by incorporating more prior information into the mcERP algorithm by adopting a sparse prior on the coupling matrix to enforce spatial localization of the sources. Such a prior would also be able to offset the effects of real variations in the recording apparatus such as impedance mismatches between electrodes. Lastly, we currently employ a crude and rather subjective stopping criterion, which could be substantially improved by deriving a criterion that is based on the principles of nodel selection. This would help to avoid possibly both overand under-fitting of the data. Regardless, the mcERP algorithm is performing as expected and the resu ts of these simulations give us confidence the mcERP algorithm will produce viable re sults when applied to real data.

\section{ACKNOWLEDGMENTS}

We would like to thank Betty Diamond, director of the Medical Scientist Training Program at the Albert Einstein College of Medicine, for her support. We are also grateful to Mitchell Steinschneider, Alan Finkelstein, and Don Faber for their helpful discussions and comments about the mcERP model and algorithm. Finally, we would like to thank Jonathan Victor and his lab for their insights and suggestions. 


\section{REFERENCES}

1. Truccolo W.A., Ding V., Knuth K.H., Nakamura R., and Bressler S.L., "Trial-to-trial Variability of Cortical Evoked Resfonses: Implications for the Analysis of Functional Connectivity," Clinical Neurophysiology, 113 2), 206-26 (2002a).

2. Schroeder C.E., Meht. A.D., and Givre S.J., "A Spatiotemporal Profile of Visual System Activation Revealed by Current Source Density Analysis in Awake Macaque," Cerebral Cortex, 8(7), 575-92 (1998).

3. Nowak, L.G. and Bull er J., "The Timing of Information Transfer in the Visual System," in Cerebral Cortex, vol 12, edited oy Rockland et al., New York: Plenum Press, 1997, pp. 205-241.

4. Schmolesky, M.T., W ang Y., Hanes D.P., Thompson K.G., Leutgeb S., Schall J.D., and Leventhal A.G., "Signal Timing Across the Macaque Visual System," Journal of Neurophysiology, 15(6), I107-18 (1998).

5. Sivia D.S., Data Analy sis. A Bayesian Tutorial, Oxford: Clarendon Press, 1996.

6. Jaynes E.T. unpublish'd. Probability Theory - The Logic of Science, http://bayes.wustl.edu.

7. Knuth K.H., "Bayesi:n source separation and localization", in Proceedings of SPIE: Bayesian Inference for Inverse F'roblems, vol. 3459, edited by A. Mohammad-Djafari, 1998, pp. 147-58.

8. Knuth K.H. and Vaug zan, H.G. Jr., "Convergent Bayesian formulations of blind source separation and electromagnetic source estimation", in Maximum Entropy and Bayesian Methods, Garching, Germany 1998, edited by W. von der Linden, V. Dose, R. Fischer, and R. Preuss, Dordrecht: Kluwer Academic Publishers, 1999, 217-26.

9. Amari S., Cichocki, A. and Yang H.H., "A New Learning Algorithm for Blind Signal Separation", in Advances in Neural Information Processing Systems 8, edited by D. Touretzky, M. Mozer, and M. Hasselmo, Cambridge. MA: MIT Press, 1996, pp. 752-763.

10. Knuth K.H., Shah A.y., Truccolo W.A., Ding M., Bressler S.L., and Schroeder C.E., "Multiple Component Event-Relıted Potential (mcERP) Estimation using Differential Amplitude and Latency Variability," in preparction.

11.Knuth K.H., Truccol), W.A., Bressler, S.L., and Ding M. "Separation of Multiple Evoked Responses Using Differential Amplitude and Latency Variability," in Proceedings of the Third International Worksh $p$ on Independent Component Analysis and Blind Signal Separation: ICA 200I, San Diego CA, 001.

12.Truccolo W.A., Knuth K.H., Ding M., and Bressler S.L. "Bayesian estimation of amplitude, latency and waveform of single trial cortical evoked components," in Maximum Entropy and Bayesian Methods, Baltimore 260l, edited by R.L. Fry and M. Bierbaum, Melville, NY: American Institute of Physics, 2002b, pp. 64-72. 